\title{
Tres textos en diálogo con la teoría del análisis didáctico de Luis Rico
}

\author{
Luis Puig, Universitat de València Estudi General
}

En este monográfico, presentamos tres textos que, con motivo de la jubilación del profesor Luis Rico, dialogan con su concepto de "análisis didáctico".

En las teorías, los términos propios de ellas suelen estar definidos para perfilar su significado, en especial cuando se está usando un término que ya tiene un campo semántico más o menos grande en su uso civil o en otras teorías, como es el caso de "análisis" y de "didáctico". En el caso de los términos propios de las teorías puede pues relajarse la consideración de que el significado está en el uso y quedarse uno con lo que diga la definición que da quien elabora la teoría -en todo caso, la definición explícita es uno de los lugares en que el término se usa. Otra cuestión sería examinar cómo se usa "análisis didáctico" en los trabajos desarrollados bajo esa teoría y contrastar lo que su uso en ellos nos dice de su significado con lo que dice la definición. Pero la intención de este monográfico no ha sido la de plantear ni una exposición ni una crítica de la teoría del análisis didáctico de Luis Rico, sino la de hacerla dialogar con otras teorías que abordan su mismo campo de estudio. Una exposición metódica de la teoría se puede encontrar en Rico, Lupiáñez y Molina, Eds. (2013), y exposiciones y aplicaciones de ella en la numerosa bibliografía de Luis Rico, sus discípulos y sus colaboradores.

El diálogo entre teorías se hace posible sobre la base de algo común que permite que los términos de una teoría puedan ser comprensibles desde la otra. En el caso del "análisis didáctico", como señala el mismo Luis Rico en el capítulo segundo de Rico, Lupiáñez y Molina, Eds. (2013), existe una ya larga historia de uso, o de usos, del término en la comunidad de investigadores en didáctica de las matemáticas en España, contemporánea de la historia de la constitución de la comunidad, de los que Luis Rico apunta un buen número de apariciones en reuniones, discusiones y documentos. Esos usos civiles en la didáctica de las matemáticas son el substrato a partir del que Luis Rico ha perfilado el significado que "análisis didáctico" tiene en su teoría, y los que permiten el diálogo entre teorías.

Para un libro que coordinó Luis Rico y se publicó en 1997, escribí un texto que titulé "Análisis fenomenológico", cuyas primeras palabras eran precisamente "El análisis didáctico". Textualmente empezaba así:

El análisis didáctico de las matemáticas, esto es, el análisis de los contenidos de las matemáticas que se realiza al servicio de la organización de su enseñanza en los sistemas educativos (Puig, 1997, p. 61).

No hay ahí estrictamente una definición de "análisis didáctico de las matemáticas", sino una especificación de qué es aquello que va a ser objeto del análisis, las matemáticas, y cuál es la intención con la que el análisis se realiza, o, 
dicho de otra manera, en qué sentido se está calificando de didáctico el análisis, y se especifica que la intención del análisis es servir a la organización de la enseñanza de las matemáticas en los sistemas educativos. En mi opinión, el diálogo de los tres textos que se reúnen en este monográfico con la teoría de Luis Rico del "análisis didáctico" es posible y fructífero porque los tres entienden que, en el análisis didáctico que interesa para la didáctica de las matemáticas, lo que se analiza es precisamente el contenido matemático y la función de ese análisis es la organización de la enseñanza de las matemáticas.

Socas, Ruano y Hernández, de la Universidad de La Laguna, presentan un texto en cuyo mismo título ya figura el término "análisis didáctico", y ese término adquiere en su texto su sentido en la teoría ELOS, desarrollada por Socas (2007), en la que se consideran tres aspectos esenciales de la producción de conocimiento en una cultura determinada, que se denominan "epistemológico", "semiótico" y "fenomenológico". El análisis didáctico es aquí, es decir, en la teoría ELOS, uno de los "supuestos básicos del enfoque competencial", junto con la "Competencia Matemática Formal", la "Competencia cognitiva" y la "Competencia de Enseñanza". En el texto, el "Análisis Didáctico [...] se describe junto con la Competencia Matemática Formal" y "en ELOS, queda organizado mediante la siguiente triada: Matemáticas curriculares, analizadas desde la Competencia Matemática Formal, representaciones semióticas y dificultades, obstáculos y errores". Por otro lado, los autores ponen en diálogo de manera explícita el Análisis Didáctico tal y como está perfilado en la teoría ELOS con el de la teoría desarrollada por Luis Rico indicando que en el caso del de Luis Rico "El Análisis Didáctico lo organizan en cinco categorías [...] Análisis Conceptual, del Contenido, Cognitivo, de Instrucción y Evaluativo", y considerando que el "Análisis Didáctico que se propone en esta investigación [es decir, el propio de la teoría ELOS] también puede ser abordado desde este enfoque con las cuatro primeras categorías".

El texto de Gómez, Figueras y Contreras, de la Universitat de València Estudi General y el Centro de Investigación y de Estudios Avanzados de México, se sitúa de entrada en el marco teórico y metodológico de los Modelos Teóricos Locales (Filloy, Rojano y Puig, 2008) y especifica que un "esquema general de un estudio en el cual se pretende construir un MTL [Modelo Teórico Local] se desglosa en dos fases que corresponden, una a la descripción de modelos de enseñanza existentes en el sistema escolar, y la otra a la experimentación para la indagación sobre las relaciones entre modelos de enseñanza existentes y las actuaciones de los alumnos". Ése es uno de los distintos tipos de estudios que se han desarrollado con la teoría de los Modelos Teóricos Locales, y en el texto se da cuenta de la primera fase de la construcción de un MTL, en concreto, sobre los algoritmos de la división de fracciones. En esa primera fase de la construcción de un MTL es donde los autores del texto, siguiendo lo que es habitual en otros estudios desarrollados con esa teoría, sitúan el análisis didáctico, junto con el análisis "histórico-epistemológico de los libros de texto tanto históricos, como actuales".

Abraham Arcavi, del Instituto Weizmann de Ciencias de Israel, por su parte, desarrolla la idea de Miradas Matemáticas, con la que se refiere

a un proceso diferente de la modelización, que es asimismo un proceso fundamental relacionado con los fenómenos cotidianos. En cierto sentido, proponemos lo 'inverso' a modelar, ya que en lugar de tratarse de imponer símbolos, procesos y operaciones a las situaciones para entenderlas mejor, se trata en cambio de develar la manera que la matemática, y en particular los números, está siendo usada por otros. Esto sería una especie de precursor del aprender a leer, descifrar y conversar con un texto matemático. 
En el texto, Arcavi muestra cómo esa idea de "miradas matemáticas" está relacionada con el análisis fenomenológico, que en la teoría del Análisis Didáctico de Luis Rico es uno de sus componentes, y propone analizar desde ese punto de vista situaciones de uso de los conceptos matemáticos, en las que ese uso no es evidente, indagando en cómo están usados, con el fin de convertirlas en tareas de enseñanza.

El contenido matemático como objeto del análisis y la organización de la enseñanza de las matemáticas como intención del análisis están presentes pues en los tres textos, en los que la forma concreta en que se realiza el análisis la modula cada una de las teorías en las que los tres estudios se sitúan. Los tres textos reunidos con esta ocasión dialogan así con la forma que adopta el Análisis Didáctico de la teoría de Luis Rico, cuya definición más detallada es la siguiente:

\begin{abstract}
El análisis didáctico es un método de investigación propio [cursiva mía] de la Didáctica de la matemática. Este método tiene su fundamento en la historia, en la propia matemática, en la filosofía del conocimiento y en las disciplinas educativas. Utiliza las técnicas del análisis conceptual y del análisis de contenido. Integra los modos analíticos de examen (escrutador, reductivo e interpretativo) con procesos de síntesis complementarios. Son objeto del análisis didáctico aquellos textos, relatos y documentos, relativos a conocimientos, normas, juicios, argumentos y explicaciones vinculados con la actividad educativa propia de la comunidad de educadores matemáticos, en su sentido más amplio. Por texto entendemos un material de comunicación escrita, que debe ser leído, interpretado y entendido por personas distintas de aquella que los escribe. El análisis didáctico se ocupa de aquellos textos que surgen de los campos conceptuales de la educación matemática, organizan sus ámbitos de actuación y regulan su práctica. (Rico, Lupiáñez y Molina, Eds., 2013, p. 13)
\end{abstract}

He puesto en cursiva en esta larga cita de Luis Rico la palabra 'propio' con la intención de señalar, para cerrar mi introducción a los textos de este monográfico, que la teoría del Análisis Didáctico de Luis Rico es lo que he llamado en otra ocasión una teoría propia de la Didáctica de las Matemáticas, una teoría que toma como objeto de estudio los procesos de enseñanza y aprendizaje de las matemáticas partiendo "de lo que hay de específico en el hecho de que lo que se está aprendiendo y enseñando es precisamente matemáticas" (Puig, 2016, p. 5). El hecho de que sea una teoría propia en ese sentido también hace posible y más fructífero el diálogo.

\title{
Referencias
}

Filloy, E., Puig, L. \& Rojano, T. (2008). Educational Algebra. A Theoretical and Empirical Approach. New York: Springer.

Puig, L. (1997). Análisis fenomenológico. En L. Rico (Coord.) La Educación Matemática en la Enseñanza Secundaria (pp. 61-94). Barcelona: Horsori.

Puig, L. (2016). De nobis ipsis silemus. Conferencia invitada al Simposio Investigación en Educación Matemática. Homenaje al profesor Luis Rico. Granada, 27-29 de enero de 2016. Recuperado el 24 de marzo de 2016 de http://www.uv.es/puigl/actividadesantiguas.html

Rico, L., Lupiáñez, J. L., \& Molina, M. (2013). Análisis Didáctico en Educación Matemática. Metodología de investigación, formación de profesores e innovación curricular. Granada: Comares

Socas, M. M. (2007). Dificultades y errores en el aprendizaje de las Matemáticas. Análisis desde el Enfoque Lógico Semiótico. En Camacho, M., Flores, P., \& Bolea, P. (Eds.) Investigación en Educación Matemática XI (pp. 19-52). La Laguna: SEIEM. 
Agradecimientos. Este trabajo se ha realizado en el marco del proyecto de investigación del Ministerio de Ciencia e Innovación, referencia: EDU2012-35638.

Plan Nacional de I+D+i. 